Pacific Journal of Mathematic 


\title{
CONTINUATION OF BOUNDED HOLOMORPHIC FUNCTIONS FROM CERTAIN SUBVARIETIES TO WEAKLY PSEUDOCONVEX DOMAINS
}

\author{
KENZŌ ADACHI
}

Let $D$ be a weakly pseudoconvex domain in $C^{n}$ with $C^{\infty}$-boundary and $V$ be a subvariety in $D$ which intersects $\partial D$ transversally. If $\partial V$ is nonsingular and consists of strictly pseudoconvex boundary points of $D$, then any bounded holomorphic function in $V$ can be extended to a bounded holomorphic function in $D$.

1. Introduction. Let $\Omega$ be an open set in some complex manifold. We denote by $H^{\infty}(\Omega)$ the space of all bounded holomorphic functions in $\Omega$ and by $A(\Omega)$ the space of all holomorphic functions in $\Omega$ which are continuous in $\bar{\Omega}$. Let $G$ be a bounded strictly pseudoconvex domain in $C^{n}$ with $C^{2}$-boundary and $\tilde{M}$ be a submanifold in a neighborhood of $\bar{G}$ which intersects $\partial G$ transversally. Let $M=\tilde{M} \cap G$. Then Henkin [5] proved the following.

FundAMENTAL THEOREM. There exists a continuous linear operator

$$
E: H^{\infty}(M) \rightarrow H^{\infty}(G) \text { satisfying }\left.E f\right|_{M}=f .
$$

Moreover $E f \in A(G)$ if $f \in A(M)$.

In the present paper we shall extend the above results to the weakly pseudoconvex case. Let $D$ be a bounded weakly pseudoconvex domain in $C^{n}$ with $C^{\infty}$-boundary. Let $\tilde{V}$ be a subvariety in a neighborhood $\tilde{D}$ of $\bar{D}$ which intersects $\partial D$ transversally. Let $V=\tilde{V} \cap D$ and $D=\{z \in \tilde{D}$ : $\rho(z)<0\}$. Suppose that $\tilde{V}$ is written in the form

$$
\tilde{V}=\left\{z \in \tilde{D}: h_{1}(z)=\cdots=h_{p}(z)=0\right\},
$$

where $h_{1}, \ldots, h_{p}$ are holomorphic in $\tilde{D}$ and $\partial h_{1} \wedge \cdots \wedge \partial h_{p} \neq 0$ on $\partial D \cap \tilde{V}$. In addition, we assume that $\partial V$ consists of strictly pseudoconvex boundary points of $D$. In this setting we shall show the following:

\section{THEOREM 1. There exists a continuous linear operator}

$$
E: H^{\infty}(V) \rightarrow H^{\infty}(D) \text { satisfying }\left.E f\right|_{V}=f .
$$

Moreover $E f \in A(D)$ if $f \in A(V)$. 
In the case when $p=1$, the above theorem is nothing but the result of Adachi [1].

I wish to thank Professor N. Kerzman who gave me the validity of Proposition 3. I also wish to thank the referee for several helpful suggestions.

2. Some results. Let

$$
s(\zeta, z)=\left(s_{1}(\zeta, z), \ldots, s_{n}(\zeta, z)\right): \partial D \times D \rightarrow C^{n}
$$

be a $C^{\infty}$ function that satisfies

$$
\langle s, \zeta-z\rangle=\sum_{j=1}^{n} s_{j}\left(\zeta_{j}-z_{j}\right) \neq 0 \quad \text { for }(\zeta, z) \in \partial D \times D .
$$

Then Hatziafratis [3] proved the following theorem.

THEOREM 2. For $f \in A(V)$ and $z \in V$ we have the integral formula

$$
f(z)=\int_{\partial V} f(\zeta) \frac{K(\zeta, z)}{\langle s, \zeta-z\rangle^{n-p}}
$$

where $K(\zeta, z)$ is a $C^{\infty}(n-p, n-p-1)$-form on $\partial D \times D$. Moreover, if $s_{1}(\zeta, z), \ldots, s_{n}(\zeta, z)$ are holomorphic in $z$, then $K(\zeta, z)$ is also holomorphic in $z$.

Let $G$ be a bounded strictly pseudoconvex domain in $C^{n}$ with $C^{\infty}$ boundary. According to the construction of Henkin [4], there exist a neighborhood $U$ of $\bar{G}$, a neighborhood $V$ of $\partial G$, and a $C^{\infty}$ function $\Phi$ : $V \times U \rightarrow C$ such that for each $\zeta \in V, \Phi(\zeta, z)$ is holomorphic in $U$ and such that $\Phi(\zeta, z)=0$ implies $\zeta=z$. Moreover, $\Phi$ admits a division

$$
\Phi(\zeta, z)=\sum_{j=1}^{n} P_{j}(\zeta, z)\left(\zeta_{j}-z_{j}\right)
$$

where $P_{j}: V \times U \rightarrow C$ of class $C^{\infty}$ and holomorphic in the second variable. In addition, if we set

$$
\begin{aligned}
T(\zeta, z)= & 2 \sum_{i=1}^{n} \frac{\partial \rho}{\partial z_{i}}(\zeta)\left(z_{i}-\zeta_{i}\right) \\
& +\sum_{i, j=1}^{n} \frac{\partial^{2} \rho}{\partial z_{i} \partial z_{j}}(\zeta)\left(z_{i}-\zeta_{i}\right)\left(z_{j}-\zeta_{j}\right)
\end{aligned}
$$


then there exists a positive constant $r$ such that

$$
\Phi(\zeta, z)=T(\zeta, z) G(\zeta, z) \text { for }\{(\zeta, z) \in V \times U:|\zeta-z|<r\}=S_{r}
$$

where $G(\zeta, z)$ is a non-vanishing $C^{\infty}$ function in $S_{r}$.

Now we have the following proposition using the techniques of the proof of Fornaess Imbedding theorem [2].

Proposition 1. Let $D$ be a bounded weakly pseudoconvex domain in $C^{n}$ with $C^{\infty}$ boundary. Let $K$ be a compact subset of $\partial D$ and consist of strictly pseudoconvex boundary points of $D$. Then there exists a strictly pseudoconvex domain $\hat{D}$ in $C^{n}$ with $C^{\infty}$ boundary such that $\hat{D} \supset D$ and $\partial \hat{D}$ coincides with $\partial D$ near $K$.

In view of Proposition 1 , if we can get the extension $F$ to $\hat{D}$, then $\left.F\right|_{D}$ is the required function. Therefore we may assume that $D$ is a strictly pseudoconvex domain. Let $\left\{\varepsilon_{\nu}\right\}$ be a sequence of positive numbers which converges to 0 . Let $D_{\nu}=\left\{z \in D: \rho(z)<-\varepsilon_{\nu}\right\}, V_{\nu}=V \cap D_{\nu}$; and $n-p=k$. If $f \in H^{\infty}(V)$, then by Hatziafratis [3], we have, for large $\nu$ and $z \in V_{\nu}$,

$$
f(z)=\int_{\partial V} f(\zeta) \frac{K(\zeta, z)}{\Phi(\zeta, z)}
$$

where $K(\zeta, z)$ is a $C^{\infty}(k, k-1)$-form depending holomorphically on $z$. We set for $z \in D_{\nu}$

$$
H_{\nu}(z)=\int_{\partial V_{\nu}} f(\zeta) \frac{K(\zeta, z)}{\Phi(\zeta, z)^{k}}
$$

Then we have the following proposition which is proved by the same argument as the proof of lemma 1 in Adachi [1].

Proposition 2. For $z \in \bar{D} \mid \partial V, H(z)=\lim _{\nu \rightarrow \infty} H_{\nu}(z)$ exists. $H(z)$ is holomorphic in D and $H(z)=f(z)$ for $z \in V$.

Let $z^{0} \in \partial V$ and $S_{z^{0}, \sigma}=\left\{z:\left|z-z^{0}\right|<\sigma\right\}$. Then there exist a constant $\sigma_{1}>0$ and a biholomorphic change of coordinates on a neighborhood of $z^{0}$ such that $\rho$ is strictly convex in a neighborhood of

$$
\bar{D} \cap S_{z^{0}, \sigma_{1}}, \quad V \cap S_{z^{0}, \sigma_{1}}=\left\{z \in S_{z^{0}, \sigma_{1}}: z_{p+1}=\cdots=z_{n}=0\right\},
$$


and $\left(\partial \rho / \partial z_{i}\right)\left(z^{0}\right) \neq 0$ for some $i(1 \leq i \leq p)$. Without loss of generality we may assume that $\left(\partial \rho / \partial z_{1}\right)\left(z^{0}\right) \neq 0$. Let $z \in S_{z_{0}^{0}, \sigma_{1}}$. We consider the system of equations for $\zeta^{0}=\left(\zeta_{1}^{0}, \ldots, \zeta_{n}^{0}\right)$ of the following form:

$$
\left\{\begin{array}{l}
\sum_{i=1}^{n} \frac{\partial \rho}{\partial \zeta_{i}}\left(\zeta^{0}\right)\left(\zeta_{i}^{0}-z_{i}\right)=0, \\
\zeta_{i}^{0}=z_{i} \quad(i=2, \ldots, p), \quad \zeta_{p+1}^{0}=\cdots=\zeta_{n}^{0}=0 .
\end{array}\right.
$$

LEMmA 1. There exist positive constants $\sigma_{2}\left(<\sigma_{1}\right), \gamma_{1}$ and $\gamma_{2}$, depending only on $D$ and $V$, such that for any $\sigma \leq \sigma_{2}$ and any $z \in S_{z^{0}, \sigma / 2}$ there exists a unique solution $\zeta^{0}=\zeta^{0}(z)$ of the system (1) which belongs to the set $S_{z^{0}, \sigma} \cap \tilde{V}$. Here the point $\zeta^{0}=\zeta^{0}(z)$ has the following properties:

(2) $\left|z-\zeta^{0}\right|^{2} \leq\left[\rho(z)-\rho\left(\zeta^{0}\right)\right] / \gamma_{1}$

(3) $\left|z-\zeta^{0}\right|^{2} \geq\left|z_{p+1}\right|^{2}+\cdots+\left|z_{n}\right|^{2} \geq \gamma_{2}\left[\rho(z)-\rho\left(\zeta^{0}\right)\right]$.

Proof. From the system (1), we have

$$
\zeta_{1}=z_{1}-\sum_{i=p+1}^{n} \frac{\partial \rho}{\partial z_{i}}(\zeta) z_{i}\left(\frac{\partial \rho(\zeta)}{\partial z_{1}}\right)^{-1} .
$$

We set

$$
a_{i}(\zeta)=-\frac{\partial \rho}{\partial z_{i}}(\zeta)\left(\frac{\partial \rho}{\partial z_{1}}(\zeta)\right)^{-1}
$$

then $a_{i}(\zeta)$ is $C^{\infty}$ in a neighborhood of $z^{0}$. We set by recurrence that

$$
\begin{aligned}
& \zeta_{1}^{(1)}=z_{1}, \zeta^{(j)}=\left(\zeta_{1}^{(j)}, z_{2}, \ldots, z_{p}, 0, \ldots, 0\right) \\
& \zeta_{1}^{(j)}=z_{1}+\sum_{i=p+1}^{n} a_{i}\left(\zeta^{(j-1)}\right) z_{i} .
\end{aligned}
$$

Then

$$
\begin{aligned}
\left|\zeta_{1}^{(j)}-\zeta_{1}^{(j-1)}\right| & \leq \sum_{i=p+1}^{n}\left|\nabla a_{i}\right|\left|\zeta_{1}^{(j-1)}-\zeta_{1}^{(j-2)}\right|\left|z_{i}\right| \\
& \leq \frac{1}{2}\left|\zeta_{1}^{(j-1)}-\zeta_{1}^{(j-2)}\right| .
\end{aligned}
$$

Therefore $\left\{\zeta^{(j)}\right\}$ converges. $\lim _{j \rightarrow \infty} \zeta^{(j)}=\zeta^{0}$ is the solution of the system (1). The strict convexity of the function $\rho$ and the equation (1) imply

(4) $\rho\left(\zeta^{0}\right)-\rho(z)+\gamma_{1}\left|\zeta^{0}-z\right|^{2} \leq 0$,

(5) $\rho\left(\zeta^{0}\right)-\rho(z)+\gamma_{2}^{\prime}\left|\zeta^{0}-z\right|^{2} \geq 0$. 
From the inequality (4), we have the inequality (2). From the system (1), we have

$$
\begin{aligned}
\left|\zeta^{0}-z\right|^{2} & =\left|z_{p+1}\right|^{2}+\cdots+\left|z_{n}\right|^{2}+\left|\zeta_{1}^{0}-z_{1}\right|^{2} \\
& \leq\left|z_{p+1}\right|^{2}+\cdots+\left|z_{n}\right|^{2}+\left(\sum_{i=p+1}^{n}\left|a_{i}\left(\zeta^{0}\right)\right|\left|z_{i}\right|\right)^{2} \\
& \leq \gamma_{2}^{\prime \prime}\left(\left|z_{p+1}\right|^{2}+\cdots+\left|z_{n}\right|^{2}\right) .
\end{aligned}
$$

Together with the inequality (5), we have

$$
\left|\zeta^{0}-z\right|^{2} \geq\left|z_{p+1}\right|^{2}+\cdots+\left|z_{n}\right|^{2} \geq \gamma_{2}\left(\rho(z)-\rho\left(\zeta^{0}\right)\right) .
$$

This completes the proof of Lemma 1.

3. Proof of Theorem 1. At first we prove that if $f \in H^{\infty}(V)$, then $H(z) \in H^{\infty}(D)$. Let $z \in S_{z^{0}, \sigma_{1}} \cap D_{\nu}$. We set

$$
\tilde{H}_{\nu}(z)=\int_{\partial V_{\nu} \cap S_{z^{0}, \sigma_{1}}} \frac{f(\zeta) K(\zeta, z)}{\Phi(\zeta, z)^{k}} .
$$

It is sufficient to show that

$$
\left|\tilde{H}_{\nu}(z)\right| \leq \gamma_{3} \sup _{\zeta \in V}|f(\zeta)| .
$$

Lemma 2. Let $f(z) \in H^{\infty}(V)$. Then for any point $z^{0} \in \partial V$ and any point $z \in \partial\left(S_{z^{0}, \sigma} \cap D_{\nu}\right) \mid \partial V_{\nu},\left(\sigma<\sigma_{2} / 2\right)$, we have

$$
\left|\frac{d \tilde{H}_{\nu}\left(\zeta^{0}+\lambda\left(z-\zeta^{0}\right)\right)}{d \lambda}\right|_{\lambda=1}\left|\leq \gamma_{4} \sup _{\zeta \in V}\right| f(\zeta) \mid .
$$

Proof of Lemma 2. We set $\varepsilon=\left(\left|z_{p+1}\right|^{2}+\cdots+\left|z_{n}\right|^{2}\right)^{1 / 2}$, where $z=$ $\left(z_{1}, \ldots, z_{n}\right) \in \partial\left(S_{z^{0}, \boldsymbol{\sigma}} \cap D_{\nu}\right) \mid \partial V_{\nu}$. By Lemma 1 , we have

$$
\varepsilon \leq\left|\zeta^{0}-z\right| \leq\left\{\frac{\rho(z)-\rho\left(\zeta^{0}\right)}{\gamma_{1}}\right\}^{1 / 2} \leq \frac{\varepsilon}{\left(\gamma_{1} \gamma_{2}\right)^{1 / 2}} .
$$

Since

$$
\sum_{i=1}^{n} \frac{\partial \rho}{\partial \zeta_{i}}\left(\zeta^{0}\right)\left(\zeta_{i}^{0}-z_{i}\right)=0
$$


it follows that

$$
\left|\sum_{i=1}^{n} \frac{\partial \Phi}{\partial z_{i}}(\zeta, z)\left(\zeta_{i}^{0}-z_{i}\right)\right| \leq \gamma_{5} \varepsilon(|\zeta-z|+\varepsilon) .
$$

On the other hand, we have

$$
\begin{aligned}
\left.\frac{d \tilde{H}_{\nu}(z+\lambda w)}{d \lambda}\right|_{\lambda=0}= & \int_{\partial V_{\nu} \cap S_{z^{0}, \sigma_{1}}} \frac{f(\zeta) \sum_{j=1}^{n} \frac{\partial K}{\partial z}(\zeta, z) w_{j}}{\Phi(\zeta, z)^{k}} \\
& -\int_{\partial V_{\nu} \cap S_{z^{0}, \sigma_{1}}} \frac{f(\zeta) K(\zeta, z) k \sum_{j=1}^{n} \frac{\partial \Phi}{\partial z_{j}}(\zeta, z) w_{j}}{\Phi(\zeta, z)^{k+1}}
\end{aligned}
$$

Therefore we have

$$
\begin{aligned}
\left|\frac{d \tilde{H}_{\nu}\left(\zeta^{0}+\lambda\left(z-\zeta^{0}\right)\right)}{d \lambda}\right|_{\lambda=1} \mid \leq & \gamma_{6} \int_{\partial V_{\nu} \cap S_{z^{0}, \sigma_{1}}} \frac{\varepsilon|f(\zeta)| d \lambda}{|\Phi(\zeta, z)|^{k}} \\
& +\gamma_{7} \int_{\partial V_{\nu} \cap S_{z^{0}, \sigma_{1}}} \frac{|f(\zeta)| \varepsilon(|\zeta-z|+\varepsilon) d \lambda}{|\Phi(\zeta, z)|^{k+1}}
\end{aligned}
$$

where $d \lambda$ is surface measure on $\partial V_{\nu}$. We can choose coordinates $\left(\eta_{1}(\zeta), \ldots, \eta_{n}(\zeta)\right)$ in $S_{z^{0}, \sigma_{2}}$ such that

$$
\eta_{1}(\zeta)=\rho(\zeta)-\rho(z)+i \operatorname{Im} \Phi(\zeta, z)
$$

Then we have

$$
|\Phi(\zeta, z)| \geq \gamma_{8}\left[\left(t_{1}+|\zeta-z|^{2}\right)^{2}+t_{2}^{2}\right]^{1 / 2}
$$

By the estimates of Henkin [5], we have

$$
\left|\frac{d \tilde{H}_{\nu}\left(\zeta^{0}+\lambda\left(z-\zeta^{0}\right)\right)}{d \lambda}\right|_{\lambda=1}\left|\leq \gamma_{9} \sup _{\zeta \in V}\right| f(\zeta) \mid .
$$

This completes the proof of Lemma 2.

By the same method as the proof of Henkin [5] (cf. Adachi [1]), we can prove that

$$
\sup _{z \in D}|H(z)| \leq \gamma_{10} \sup _{\zeta \in V}|f(\zeta)| .
$$


The next step is to show that if $f \in A(V)$, the $H(z) \in A(D)$. In order to prove this statement, we need the following modified version of $\mathrm{N}$. Kerzman [6]. In the Theorem 1.4.1' of Kerzman, $V$ is a manifold. But the proof of the theorem is applicable in our case.

Proposition 3. Let $f \in A(V)$. Then there exists a sequence $\left\{f_{k}\right\}$ of holomorphic functions in a neighborhood of $\bar{V}$ in $\tilde{V}$ such that $\left\|f_{k}-f\right\|_{V} \rightarrow 0$ when $k \rightarrow \infty$.

From Proposition 3 we can suppose that $f$ is holomorphic in $\bar{V}^{\prime}$ $\left(V \subset V^{\prime} \subset \bar{V}^{\prime} \subset \tilde{V}\right)$. Let $z^{0} \in \partial V$ and let $z \in S_{z^{0}, \sigma / 2} \cap\left(\bar{D}_{\nu} \mid \partial V_{\nu}\right)$. By using Stokes' formula, we have

$$
\begin{aligned}
H_{\nu}(z)= & \int_{\partial V^{\prime}} \frac{f(\zeta) K(\zeta, z)}{\Phi(\zeta, z)^{k}}-\int_{\left(V^{\prime}-V_{\nu}\right) \cap S_{z^{0}, 2 \sigma}} f(\zeta) \bar{\partial}_{\zeta}\left(\frac{K(\zeta, z)}{\Phi(\zeta, z)^{k}}\right) \\
& -\int_{\left(V^{\prime}-V_{\nu}\right) \mid S_{z_{0}, 2 \sigma}} f(\zeta) \bar{\partial}_{\zeta}\left(\frac{K(\zeta, z)}{\Phi(\zeta, z)^{k}}\right) .
\end{aligned}
$$

Therefore it is sufficient to show that

$$
F_{\nu}(z)=\int_{\left(V^{\prime}-V_{\nu}\right) \cap S_{z^{0}, 2 \sigma}} f(\zeta) \bar{\partial}_{\zeta}\left(\frac{K(\zeta, z)}{\Phi(\zeta, z)^{k}}\right)
$$

is continuous at $z^{0}$. In order to prove this fact, we need the following.

Lemma 3. Let $z \in S_{z^{0}, \sigma / 2} \cap\left(\bar{D}_{\nu} \mid \partial V_{\nu}\right)$. Then it follows that

$$
\left|\frac{d F_{\nu}\left(\zeta^{0}+\lambda\left(z-\zeta^{0}\right)\right)}{d \lambda}\right|_{\lambda=1}\left|\leq \gamma_{11} \varepsilon\right| \log \varepsilon\left|\sup _{\zeta \in V}\right| f(\zeta) \mid,
$$

where $\zeta^{0}=\zeta^{0}(z)$ is the solution of the system (1).

Proof of Lemma 3. We can write

$$
\begin{aligned}
F_{\nu}(z)= & \int_{\left(V^{\prime}-V_{\nu}\right) \cap S_{z^{0}, 2 \sigma}} f(\zeta) \frac{A(\zeta, z)}{\Phi(\zeta, z)^{k}} \\
& +\int_{\left(V^{\prime}-V_{\nu}\right) \cap S_{z^{0}, 2 \sigma}} \frac{f(\zeta) \sum_{j=1}^{n}\left(\zeta_{j}-z_{j}\right) B_{j}(\zeta, z)}{\Phi(\zeta, z)^{k+1}}
\end{aligned}
$$


where $A(\zeta, z)$ and $B_{j}(\zeta, z)$ are $(k, k)$-forms which are smooth in $(\zeta, z)$ and holomorphic in $z$. Therefore

$$
\begin{aligned}
\left|\frac{d F_{\nu}\left(\zeta^{0}+\lambda\left(z-\zeta^{0}\right)\right)}{d \lambda}\right|_{\lambda=1} \mid & \\
\leq & \gamma_{12} \int_{\left(V^{\prime}-V_{\nu}\right) \cap S_{z^{0}, 2 \sigma}}|f(\zeta)| \frac{\varepsilon d \lambda}{|\Phi(\zeta, z)|^{k+1}} \\
& +\gamma_{13} \int_{\left(V^{\prime}-V_{\nu}\right) \cap S_{z^{0}, 2 \sigma}} \frac{|f(\zeta)||\zeta-z| \varepsilon(|\zeta-z|+\varepsilon) d \lambda}{|\Phi(\zeta, z)|^{k+2}} .
\end{aligned}
$$

By applying the estimates of Henkin [5], we have the inequaltiy (6). This completes the proof of Lemma 3.

Using the method of Henkin [5], we can prove

$$
\left|F_{\nu}(z)-F_{\nu}\left(z^{0}\right)\right| \leq \gamma_{14} \sigma|\log \sigma| \sup _{\zeta \in V^{\prime}}|f(\zeta)|+\sigma \sup _{\zeta \in V^{\prime}}|\operatorname{grad} f(\zeta)| .
$$

Therefore $F_{\nu}(z)$ is continuous at $z^{0}$. This completes the proof of Theorem 1.

\section{REFERENCES}

[1] K. Adachi, Extending bounded holomorphic functions from certain subvarieties of a weakly pseudoconvex domain, Pacific J. Math., 110, No. 1 (1984), 9-19.

[2] J. E. Fornaess, Embedding strictly pseudoconvex domains in convex domains, Amer. J. Math., 98 (1976), 529-569.

[3] T. E. Hatziafratis, Integral representation formulas on analytic varieties, Pacific J. Math., 123, No. 1 (1986), 71-91.

[4] G. M. Henkin, Integral representations of functions holomorphic in strictly pseudoconvex domains and some applications, Math. USSR Sbornik, 7 (1969), 597-616.

[5] Continuation of bounded holomorphic functions from submanifolds in general position to strictly pseudoconvex domains, Izv. Akad. Nauk SSSR, 36 (1972), 540-567.

[6] N. Kerzman, Hölder and $L^{p}$-estimates for solutions of $\bar{\partial} u=f$ in strongly pseudoconvex domains, Comm. Pure Appl. Math., 24 (1971), 301-380.

Received July 2, 1986.

NAGASAKI UNIVERSITY

NAGASAKI 852

JAPAN 


\section{PACIFIC JOURNAL OF MATHEMATICS EDITORS}

V. S. VARADARAJAN (Managing Editor) University of California Los Angeles, CA 90024 HERBERT ClEMENS University of Utah Salt Lake City, UT 84112

R. FINN

Stanford University

Stanford, CA 94305

\section{HERMANN FLASCHKA} University of Arizona Tucson, AZ 85721

RAMEsh A. GANGolli University of Washington Seattle, WA 98195

VAUghan F. R. JONES

University of California Berkeley, CA 94720

\section{ROBION KIRBY}

University of California

Berkeley, CA 94720

C. C. MOORE

University of California

Berkeley, CA 94720

HAROLD STARK

University of California, San Diego

La Jolla, CA 92093

\section{ASSOCIATE EDITORS}
R. ARENS
E. F. BECKENBACH
B. H. NEUMANN
F. WOLF
K. YOSHIDA (1906-1982)

\section{SUPPORTING INSTITUTIONS}

UNIVERSITY OF ARIZONA

UNIVERSITY OF BRITISH COLUMBIA

CALIFORNIA INSTITUTE OF TECHNOLOGY

UNIVERSITY OF CALIFORNIA

MONTANA STATE UNIVERSITY

UNIVERSITY OF NEVADA, RENO

NEW MEXICO STATE UNIVERSITY

OREGON STATE UNIVERSITY
UNIVERSITY OF OREGON

UNIVERSITY OF SOUTHERN CALIFORNIA

STANFORD UNIVERSITY

UNIVERSITY OF HAWAII

UNIVERSITY OF TOKYO

UNIVERSITY OF UTAH

WASHINGTON STATE UNIVERSITY

UNIVERSITY OF WASHINGTON 


\section{Pacific Journal of Mathematics}

Vol. 130, No. $1 \quad$ September, 1987

K. Adachi, Continuation of bounded holomorphic functions from certain subvarieties to weakly pseudoconvex domains $\ldots \ldots \ldots \ldots \ldots \ldots \ldots$

Erazm Jerzy Behr, Enveloping algebras of Lie superalgebras ........... 9

Dong M. Chung, Scale-invariant measurability in abstract Wiener spaces . . . 27

Peter Gerard Dodds and Bernardus de Pagter, Algebras of unbounded

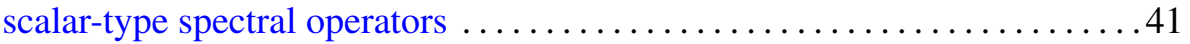

Wu-Yi Hsiang and Hsueh-Ling Huynh, Generalized rotational

hypersurfaces of constant mean curvature in the Euclidean spaces. II . . . .75

Harvey Bayard Keynes and M. Sears, Time changes for $\mathbf{R}^{n}$ flows and suspensions ..................................... 97

Frances Kirwan, Ronnie Lee and Steven Howard Weintraub, Quotients

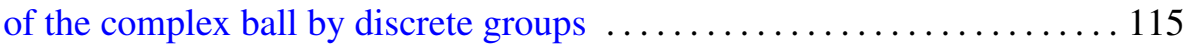

Magnhild Lien, Groups of knots in homology 3-spheres that are not

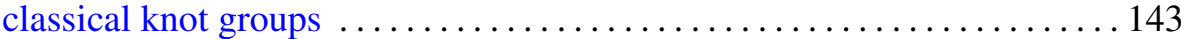

Juan Carlos Migliore, Liaison of a union of skew lines in $\mathbf{P}^{4} \ldots \ldots \ldots \ldots 153$

Jesper M. Møller, Spaces of sections of Eilenberg-Mac Lane fibrations . . . . 171 Daniel Ullman, A generalization of a theorem of Atkinson to noninvariant

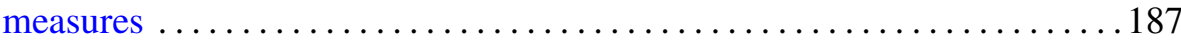

Kohhei Yamaguchi, Operations which detect $\mathscr{P}^{1}$ in odd primary connective



